\title{
The Mid-infrared Spectral Properties of Starburst Galaxies and Active Galactic Nuclei
}

\author{
O. Laurent and I.F. Mirabel \\ CEA/DSM/DAPNIA. Service d'Astrophysique F-91191 Gif-sur-Yvette, \\ France
}

\begin{abstract}
.
We present a new diagnostic tool based on mid-infrared spectra (5$16 \mu \mathrm{m})$ for distinguishing the emission triggered by the active galactic nucleus (AGN) and by the star formation activity. We show that the AGN spectra, contrary to the starburst spectra, present an important continuum below $9 \mu \mathrm{m}$ and an absence of Unidentified Infrared Bands (UIBs).
\end{abstract}

\section{Introduction}

Nuclear regions harboring starbursts or AGNs are usually embedded in a large concentration of dust producing heavy obscuration especially for interacting galaxies which contain a large amount of molecular gas in their inner regions (see Sanders \& Mirabel 1996 for a review). Since large amounts of molecular gas are needed for fueling nuclear starbursts as well as AGNs, the absorption makes the distinction between starburst and AGN difficult. Mid-IR wavelengths, being less affected by the absorption $\left(A_{V} / A_{8 \mu m} \sim 50\right)$, can probe obscured regions usually not visible in optical and near-IR wavelengths as well as the re-radiated emission from dust (Mirabel et al. 1998a, 1998b). We present a statistical approach to classify the spectral diversity of a large sample of galaxies observed in mid-IR wavelengths.

\section{Observations}

Based on the spectral-imaging capabilities of the Infrared Space Observatory (ISO) ${ }^{4}$, a sample of 25 active/interacting galaxies was observed with the midinfrared camera on-board ISO (Cesarsky et al. 1996a). Standard data reduction techniques were applied using the CAM Interactive Analysis software $(\mathrm{CIA})^{5}$.

\footnotetext{
${ }^{4}$ Based on observations with ISO, an ESA project with instruments funded by ESA Member States (especially the PI countries : France, Germany, the Netherlands and the United Kingdom) and with participation of ISAS and NASA.

${ }^{5} \mathrm{CIA}$ is a joint development by the ESA astrophysics division and the ISOCAM consortium led by the ISOCAM PI, C. Cesarsky, Direction des Sciences de la Matière, C.E.A. France.
} 

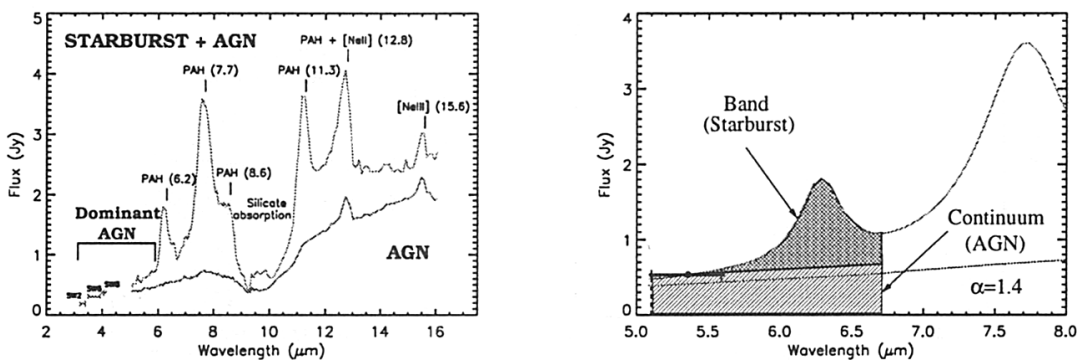

Figure 1. Left panel: Mid-IR spectra of NGC 5128 with the lower curve for the nuclear region (100pc size) and the upper curve for a larger integrated region ( $800 \mathrm{pc}$ size). The AGN spectrum represents $45 \%$ of the energy between $5 \mu \mathrm{m}$ and $16 \mu \mathrm{m}$ and it highly dominates at short wavelengths below the UIB at $6.2 \mu \mathrm{m}$ for which the emission associated with starburst activity is not detected with the three narrow band filters below $5 \mu \mathrm{m}$. Right panel: the spectrum between $5.1 \mu \mathrm{m}$ and $6.7 \mu \mathrm{m}$ is divided into two different parts. The flux above the thick line (power law with a spectral index $\alpha \sim 1.4$ fitting the AGN spectrum continuum and rescaled to the average flux between $5.1 \mu \mathrm{m}$ and $5.6 \mu \mathrm{m}$ ) estimates the flux arising from the UIB at $6.2 \mu \mathrm{m}$ and traces the starburst activity. The flux below the line selects the continuum arising mainly from the AGN. The band/continuum indicator varies similarly to the starburst/AGN ratio.

\section{Mid-IR features of AGN and Starburst spectra}

We observed nearby prototypical active galaxies (NGC 5128, NGC 1068) in order to separate with the highest spatial resolution $\left(\sim 100-400 \mathrm{pc}\right.$ with $\left.5^{\prime \prime}\right)$ the distinct mid-IR emission produced by the AGN from that produced by the circumnuclear starburst. Using observations of nearby star forming galactic regions (NGC 7023, M17, Cesarsky et al. 1996b, 1996c), we identified a typical mid-IR spectrum of a pure ionized region (HII) heated by young stars and a spectrum characterizing photo-dissociation regions (PDRs) surrounding HII regions. Those two spectra were used to separate into two components the emission from larger unresolved star forming regions which can be interpreted as a mixing of several PDRs and HII regions. Based on these results, we are able to estimate the relative contribution of each component to the mid-IR emission of unresolved regions observed in galaxies (see also Tran 1998).

\subsection{Diagnostics for distinguishing AGN, HII and PDR emission}

The UIBs, the carbonaceous species that have been called polycyclic aromatic hydrocarbons (PAHs) seen at $6.2,7.7,8.6,11.3$ and $12.8 \mu \mathrm{m}$, are detected mainly in PDRs, whereas a noticeable continuum at short wavelengths $[5-9 \mu \mathrm{m}]$ without UIBs is present in our sample of AGN spectra. Presumably, the UIBs are destroyed in AGNs by the intense radiation field. We estimate the PDRs/AGN fraction with the $\operatorname{UIB}(6.2 \mu \mathrm{m}) /$ continuum $[5-6.7 \mu \mathrm{m}]$ ratio described in Figure 1 (see also Genzel et al. 1998). 

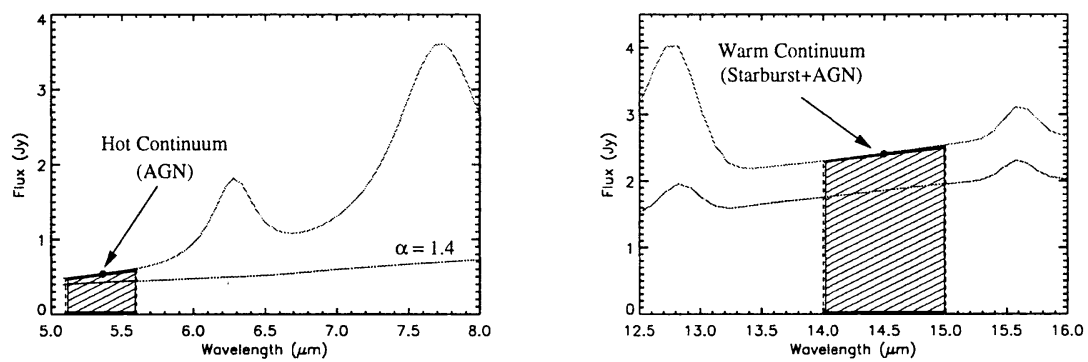

Figure 2. Left panel: The hot continuum $[5.1-5.6 \mu \mathrm{m}]$ originates mainly from the underlying emission of the AGN. Right panel: Both star forming regions and the AGN contribute to the warm continuum[14-15 $\mu \mathrm{m}]$. The ratio of the warm over hot continuum can be used to differentiate between strong starburst spectra with faint UIBs and AGN spectra.

Since the HII region spectrum has very faint thermal emission at short wavelengths compared to that of an AGN, the warm continuum[14-15 $\mu \mathrm{m}] /$ hot continuum $[5-5.6 \mu \mathrm{m}]$ ratio can be used as an estimator of the HII/AGN fraction (see Figure 2).

\subsection{Mid-IR diagram}

In Figure 3, we present the three mid-IR spectra used as templates for identifying different components associated with AGNs or starbursts. The HII regions have absent or faint UIBs and a strong rising continuum associated with strong emission lines of [ArIII] $(9 \mu \mathrm{m})$, [SIV] $(10.5 \mu \mathrm{m})$, [NeII] $(12.8 \mu \mathrm{m})$ and [NeIII] $(15.5 \mu \mathrm{m})$. The regions dominated by PDRs, observed in galactic discs, present strong UIBs largely dominating the total emission and a faint continuum. Finally, the AGN mid-IR emission is mainly characterized by strong continuum at $5-9 \mu \mathrm{m}$ without UIBs. Assuming that the spectra integrated over the whole galaxy are composed of the mixing of spectra originating from HII regions, PDRs and an AGN, we have built composite spectra from the three mid-IR templates. This diagram can be decomposed in three different regions where we select preferentially the AGN (bottom left), the HII region (top) and the PDR spectra (bottom right).

In Figure 4, we have built the same diagram including 34 spectra of galaxies containing star forming regions and/or AGNs. The different galaxies are placed in the diagram. The extreme starburst galaxies like Arp 220 and Mrk 171(A) are classified as galaxies dominated by strong star forming activity presenting a large fraction of pure HII regions compared to PDRs. The AGNs detected for nearby objects are located in the bottom left part of the diagram. The star forming regions observed in galactic discs are situated near the region dominated by PDRs.

Using this diagram, we conclude that the well-known ultraluminous galaxies like Arp 220, IRAS 23128-5919 and NGC 6240 present mid-IR spectra dominated by strong starburst activity. The AGN contribution is only detected in nearby objects for which we are able to separate the nuclear regions and the galactic discs. 

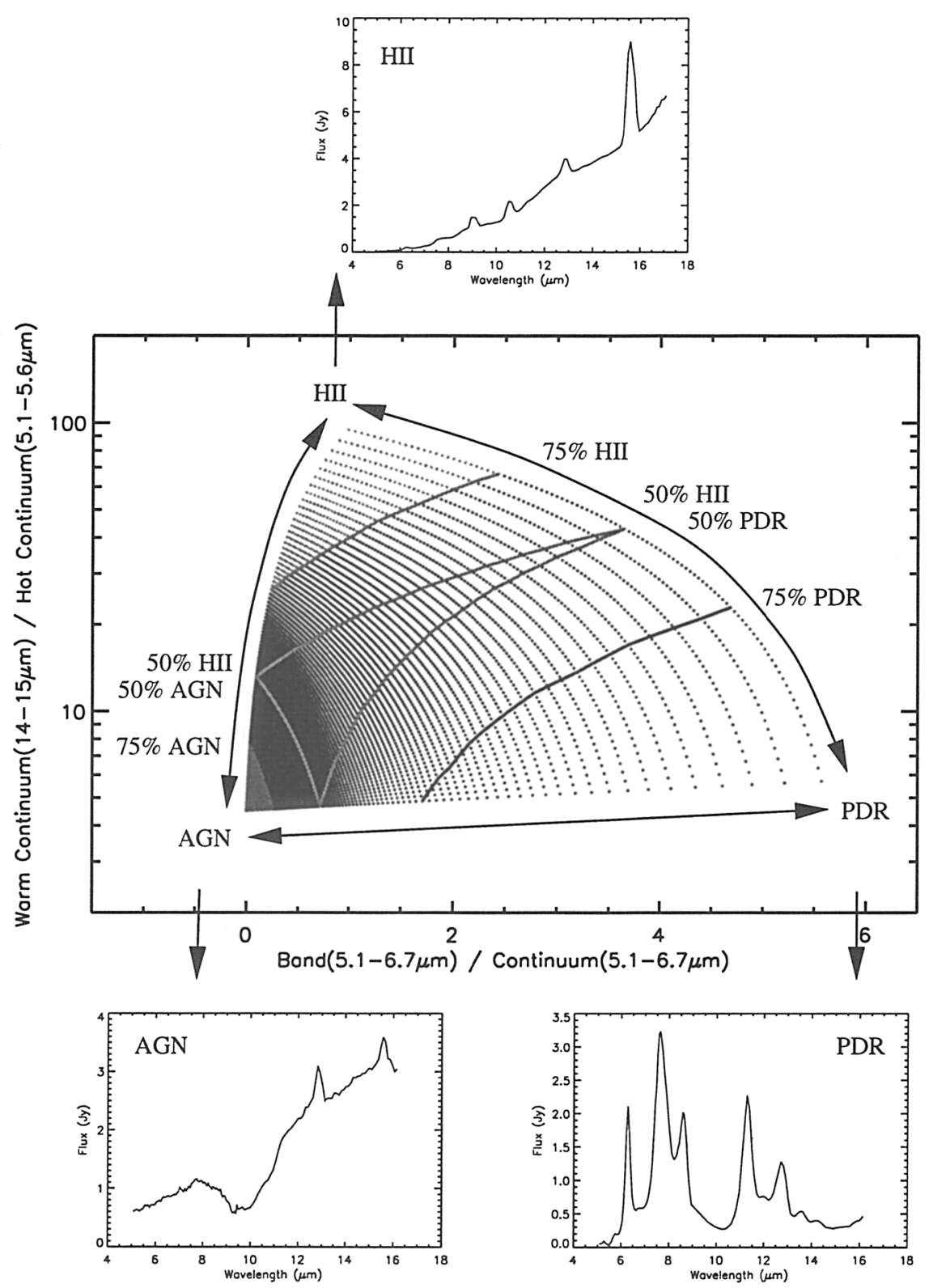

Figure 3. Based on synthetic spectra containing variable fractions of three mid-IR templates observed in a pure HII region (M17), a PDR region (NGC 7023 ) and an AGN (NGC 5128), the diagnostic diagram defines three distinct areas. In the upper part, we select spectra dominated by HII regions (strong starbursts). On the left, the AGN spectra are dominant in a very small region, and finally, the PDR spectra fall in the right part. The percentages indicate the fraction of each mid-IR contribution used in the composite spectra. 


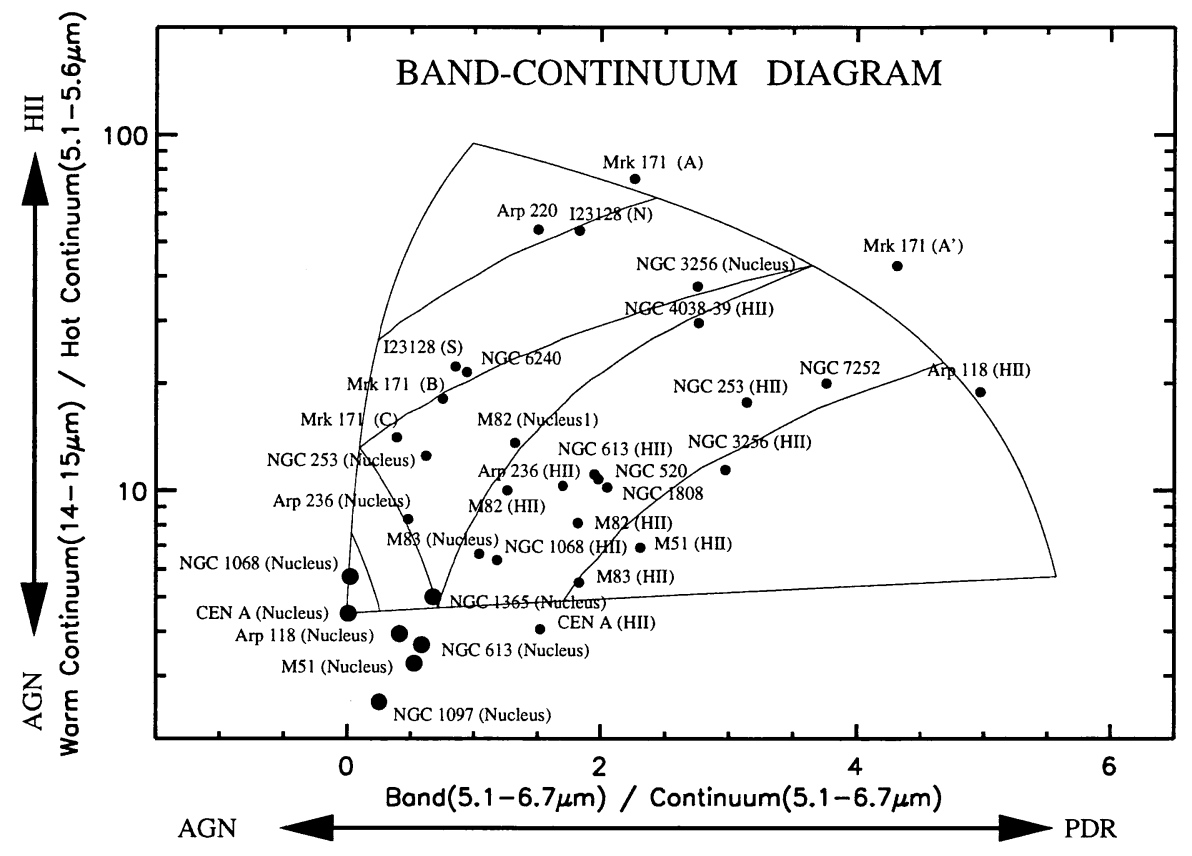

Figure 4. Diagnostic diagram including 34 spectra covering a large sample of mid-IR emission (nulcear regions, disc starburst regions called HII and unresolved galaxies). The galaxies hosting an intense starburst are in the upper part. The galactic central regions (size of several kpc) hosting an AGN (big circles) are all located in the bottom left part of the diagram. The spectra of galactic discs lie near the PDR region. The curves demarcate the AGN, PDR and HII regions according to figure 3 . 


\section{Conclusion}

We have obtained a new AGN/Starburst diagnostic using the UIB at $6.2 \mu \mathrm{m}$ and the mid-IR continuum based on two independent points:

1) The presence of a non-negligible continuum below $9 \mu \mathrm{m}$ in AGN spectra which we attribute to hot dust emission directly heated by the central engine.

2) The absence in "pure" AGN spectra of UIBs whose carriers are presumably destroyed by the strong UV-X radiation field.

Acknowledgments. We thank V. Charmandaris, H. Roussel and D. Tran for providing data in advance of publication as well as P. Gallais and S. Madden for their help on different aspects of this work.

\section{References}

Cesarsky C., Abergel A., Agnese P. et al. 1996a, A\&A, 315, L32

Cesarsky D., Lequeux J., Abergel A. et al. 1996b, A\&A, 315, L305

Cesarsky D., Lequeux J., Abergel A. et al. 1996c, A\&A, 315, L309

Genzel R., Lutz D., Sturm E. et al. 1998, Ap.J., , 498, 579

Mirabel I.F., Vigroux L., Charmandaris V. et al. 1998a, A\&A, 333, L1

Mirabel I.F., Laurent O., Sanders D.B. et al. 1998b, A\&A, in press

Sanders D.B., \& Mirabel I.F. 1996, ARA\&A, 34, 749

Tran D. 1998, PhD thesis, University of Paris XI 